\title{
The Effect of Holmium Laser Fiber Bending Radius on Power Delivery During Flexible Ureteroscopy
}

\author{
Dylan Heckscher, AB, ${ }^{1}$ Junjie Zeng, BS, ${ }^{2}$ Panagis Samolis, BS, ${ }^{2}$ Michelle Y. Sander, PhD, ${ }^{2,3}$ \\ Shaun E. Wason, MD, ${ }^{4}$ and David S. Wang, MD ${ }^{4}$
}

\begin{abstract}
Introduction: Flexible ureteroscopy is a mainstay of upper urinary tract stone treatment. Holmium laser lithotripsy is a particularly common and notable technique for the dusting and fragmenting of renal stones. During ureteroscopy, optical fibers are subject to sharp bends in pursuit of stones, particularly those at the lower pole. Following from principles of fiber optics, subjecting these fibers to sharp bending angle has the potential to reduce the efficiency of power transmission at the fiber tip. Due to the potential implications this hypothesis could have on endourological practice and research, we aimed to explore the potential impact of fiber bending on end-fiber power output.

Materials and Methods: Using a highly sensitive oscilloscope and a urological holmium laser, we assessed the end-fiber power output under a variety of bending conditions. To ensure maximal confidence in our results, the maximal bending conditions explored substantially exceeded any condition, which could occur during ureteroscopic surgery.

Results: We found evidence that bending radius alone has a clinically insignificant impact on the light power transmission in the fiber. At certain bending conditions, we observed a clinically unimportant but statistically significant reduction in power transmission. This was verified using two commonly used delivery fiber types exposed to 8-second bursts for each bending condition.
\end{abstract}

Keywords: laser, urolithiasis, kidney stone, bending radius, ureteroscopy, lithotripsy

\section{Introduction}

$\mathbf{U}$ RETEROSCOPY IS THE MAINSTAY of treatment for urinary stones in the upper urinary tract. Although the use of laser technology in urology was first documented by Parsons and colleagues in 1966, it was not until more recent decades that laser lithotripsy has become the standard for ureteroscopic management of urinary tract stones. ${ }^{1,2}$

During flexible ureteroscopy, delivery fibers may be subject to multiple sharp bends depending on stone location. Following from the principles of fiber optics, a hypothesis exists that there is a meaningful difference in laser power output during upper and lower pole ureteroscopy due to the changes in fiber positioning and bending. There is existing literature aimed to address the relationship of laser firing under sharp bending conditions rather than the potential impact of mechanical bending alone. ${ }^{3-5}$ However, there is little literature isolating the issue of mechanical bending, and none which adequately explores this question as it relates to holmium lasers.
If a substantial change in power output in different bending positions were to exist, it would affect the practice of endourology by requiring surgeons to incorporate that element into surgical planning, especially with respect to surgical approach and laser-firing parameters. It would also lead delivery fiber manufacturers to pursue novel ways of reducing these losses under clinical conditions. Given this potentially significant impact on urological practice, we sought to evaluate whether there was a measurable decrease in the power output of a commonly used holmium laser under a variety of bending conditions.

\section{Materials and Methods}

Our experimental setup consisted of a Lumenis ${ }^{\circledR}$ (Yokneam Illit, Israel) Pulse 30H Holmium Laser, a standard mirror, a multimode collection fiber with a $400 \mu \mathrm{m}$ core diameter, a Thorlabs, Inc. (Newton, NJ) amplified silicon photodetector PDA36A, and a Tektronix ${ }^{\circledR}$ (Beaverton, OR) MSO72004C

${ }^{1}$ Department of Urology, Boston University School of Medicine, Boston, Massachusetts, USA.

${ }^{2}$ Department of Electrical and Computer Engineering, BU Photonics Center, Boston University, Boston, Massachusetts, USA.

${ }^{3}$ Division of Materials Science and Engineering, Boston University, Boston, Massachusetts, USA.

${ }^{4}$ Department of Urology, Boston Medical Center, Boston University School of Medicine, Boston, Massachusetts, USA.

Prior publication as an abstract at the American Urological Association Annual Meeting, 2019. 
Oscilloscope with the aim of accurately measuring variations in the power output. Two commonly used optical fibers used in surgical settings were employed in our trials: a SlimLine ${ }^{\mathrm{TM}}$ SIS 365 , and a SlimLine $200 \mathrm{D} / \mathrm{F} / \mathrm{L}$ from Lumenis.

For the experimental setup, the delivery fiber was directly aimed at a highly reflective surface (silver mirror) and the backscattered light was collected with a multimode fiber of $400 \mu \mathrm{m}$ core diameter. The output of the collection fiber was focused on the photodetector, which was connected to the oscilloscope. The delivery fiber and collection fiber were positioned in parallel with the aid of a metal tube slightly larger than the two fibers together. The fibers were fixed in multiple locations across the tube to prevent any motion relative to each other or the metal tube, and the tube was clamped at a fixed height beneath the water's surface and above the mirror using a burette clamp pointing downward at the silver mirror. The mirror and the fiber tips were then submerged in deionized water, used to improve the reproducibility of our results. The experimental setup is depicted in Figure 1.

The laser was fired for 8 seconds at $0.3 \mathrm{~J} / 5 \mathrm{~Hz}$ without any significant bending of the delivery fiber to establish a baseline measured power output. For subsequent test conditions, a central portion of the delivery fiber was horizontally and tightly wrapped around cylinders of varying radii, without altering the positioning of the tip of the delivery fiber or collecting fiber in the water. The power output was then recorded over 8 seconds at a sampling rate of $31.25 \mathrm{MS} / \mathrm{s}$ with the oscilloscope. The recorded peak values of each pulse were used to calculate a root-mean-square (RMS), which we used as a representative value for the collected output power.

Baselines were taken for the two different diameter delivery fibers. The smallest radii used for each fiber type are the smallest radii each fiber could reliably achieve without breaking.

\section{Results}

We report that the RMS of pulse peak power did not vary clinically significantly at any bending radius or number of turns measured within a power fluctuation range of a maximum value of $3 \%$. The standard deviation reported as vari- ation in the calculated RMS amounts to $\sim 0.5 \%$, which can be attributed to laser pulse stability and the oscilloscope measurement accuracy. Thus, variations on that order of the RMS peak power were regarded as statistically insignificant. The $365 \mu \mathrm{m}$ fiber was subject to six different bending conditions in addition to a baseline measurement. At the tightest radius measured, the $365 \mu \mathrm{m}$ fiber was measured as transmitting $1.6 \%$ less power than baseline. The $200 \mu \mathrm{m}$ fiber underwent nine bending conditions, in addition to a baseline measurement. When the $200 \mu \mathrm{m}$ fiber was coiled twice with a radius of $1.3 \mathrm{~cm}$ (the tightest radius measured), the power output was measured to be slightly higher than the baseline value.

As the multiple radius bending experiments were conducted together, slight changes in the laser output power can account for this slight increase in laser power. However, the trend of measuring reduced power with increasing radius turns remains the same. After wrapping the $200 \mu \mathrm{m}$ fiber 10 times at the smallest radius it would accept without breaking - well beyond the bending condition of any achievable clinical circumstance-the power was measured to be $2.6 \%$ lower than baseline. In none of the cases did the fiber break or degrade during the experiment.

While the bending radius for the two fibers are specified by the manufacturer as 1.4 and $0.6 \mathrm{~cm}$ for the 365 and $200 \mu \mathrm{m}$ fiber, respectively, a slight power reduction is noticeable even before those bending radii are reached. The $200 \mu \mathrm{m}$ fiber transmitted $1.6 \%$ less power at a radius as wide as $1.8 \mathrm{~cm}$. The $365 \mu \mathrm{m}$ fiber showed a statistically significant reduction$1.2 \%$-in power transmission at a radius as large as $3 \mathrm{~cm}$.

Measured power outputs are summarized in Tables 1 and 2.

\section{Discussion}

Holmium lasers have revolutionized the way in which we perform ureteroscopy and stone fragmentation for urinary tract stones. All optical fibers depend on the principle of total internal reflection, derived from Snell's law $n_{1} \sin \theta_{1}=$ $n_{2} \sin \theta_{2}$, where $n$ refers to the index of refraction of two different apposed materials and $\theta$ refers to the angle of an incident light ray relative to the normal of the interface of the two materials. As $\theta$ increases-indicating the light ray has

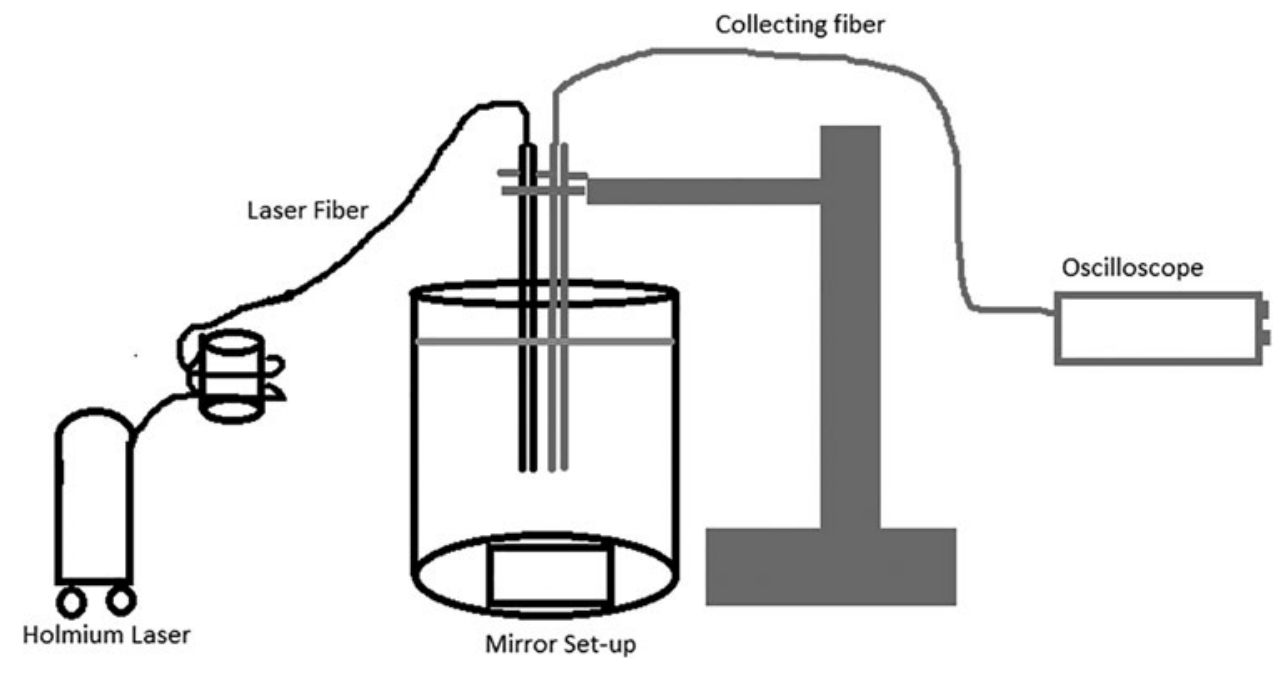

FIG. 1. The figure depicts our data-gathering laboratory set-up. The depicted laser delivery fiber was wrapped multiple times around various cylinders of recorded radius, and submerged into a beaker of deionized water pointed at a silvered mirror. A collecting fiber, linked to an oscilloscope, was inserted in parallel to the delivery fiber. 
Table 1. Power Outputs and Bending RadiI, $365 \mu \mathrm{M}$ LASER FiBER

\begin{tabular}{lcc}
\hline $\begin{array}{l}\text { Bending radius and } \\
\text { no. of turns }(R)\end{array}$ & $\begin{array}{c}\text { RMS of pulse peak } \\
\text { power }(\mathrm{au})\end{array}$ & $\begin{array}{c}\text { Standard } \\
\text { deviation }(\mathrm{au})\end{array}$ \\
\hline Baseline & 1 & 0.005 \\
$1.7 \mathrm{~cm} \mathrm{1R}$ & 0.984 & 0.005 \\
$1.9 \mathrm{~cm} \mathrm{1R}$ & 0.984 & 0.005 \\
$3 \mathrm{~cm} \mathrm{1R}$ & 0.987 & 0.005 \\
$3.5 \mathrm{~cm} \mathrm{1R}$ & 0.991 & 0.006 \\
$6 \mathrm{~cm} \mathrm{1R}$ & 0.996 & 0.004 \\
$8 \mathrm{~cm} \mathrm{1R}$ & 0.997 & 0.007 \\
\hline
\end{tabular}

This table reports the measured power output (as a RMS) of a holmium laser firing at a constant power level while its delivery fiber underwent bending of the described radii. All RMS values normalized to the baseline value. "Bending Radius" refers to the radius of the cylindrical object around which the laser delivery fiber was wrapped for one full turn. "Number of Turns" refers to the number of times the delivery fiber was fully wrapped around the cylindrical object before measurement.

$\mathrm{au}=$ arbitrary unit (normalized to 1 ); RMS = root-mean-square.

become more parallel to the interface of the two materials - it approaches and then passes a critical angle $\theta_{c}$, after which the light ray no longer undergoes refraction across the media but instead experiences total internal reflection. ${ }^{6}$

In the context of surgical fiber optics, this means that if the ray travels sufficiently parallel to the long axis of the delivery fiber, it will experience total internal reflection with minimal propagation loss. However, if the delivery fiber is bent substantially, the light may not undergo total internal reflection for two reasons. First, the incident angle may not exceed $\theta_{\mathrm{c}}$, and second, the cladding of the fiber "core" can experience microscopic deformations, which alter the value $n$.

As a result of either or both factors, the incident ray may undergo refraction instead of reflection, which translates to a loss of "modes" of light, translating to reduced light emission at the output. ${ }^{7}$ Furthermore, the penetrance of laser light into the fiber cladding can theoretically induce thermal de-

Table 2. Power Outputs and Bending Radi, $200 \mu \mathrm{M}$ LASER FiBER

\begin{tabular}{lcc}
\hline $\begin{array}{l}\text { Bending radius and } \\
\text { no. of turns }(R)\end{array}$ & $\begin{array}{c}\text { RMS of pulse peak } \\
\text { power }(\mathrm{au})\end{array}$ & $\begin{array}{c}\text { Standard } \\
\text { deviation }(\mathrm{au})\end{array}$ \\
\hline Baseline & 1 & 0.008 \\
$0.5 \mathrm{~cm} \mathrm{1R}$ & 0.978 & 0.016 \\
$1.3 \mathrm{~cm} \mathrm{1R}$ & 0.979 & 0.006 \\
$1.8 \mathrm{~cm} \mathrm{1R}$ & 0.983 & 0.006 \\
$3.4 \mathrm{~cm} \mathrm{1R}$ & 0.997 & 0.007 \\
$6 \mathrm{~cm} \mathrm{1R}$ & 0.998 & 0.007 \\
$7.6 \mathrm{~cm} \mathrm{1R}$ & 1.002 & 0.007 \\
$1.3 \mathrm{~cm} \mathrm{2R}$ & 1.020 & 0.008 \\
$1.3 \mathrm{~cm} \mathrm{5R}$ & 1 & 0.007 \\
$1.3 \mathrm{~cm} \mathrm{10R}$ & 0.974 & 0.008 \\
\hline
\end{tabular}

This table reports the measured power output (as a RMS) of a holmium laser firing at a constant power level while its delivery fiber underwent bending of the described radii. All RMS values normalized to the baseline value. "Bending Radius" refers to the radius of the cylindrical object around which the laser delivery fiber was wrapped for one full turn. "Number of Turns" refers to the number of times the delivery fiber was fully wrapped around the cylindrical object before measurement. struction of the fiber itself. ${ }^{7}$ This concept is supported by research demonstrating thermal breakdown in flexed delivery fibers during lower pole nephroscopy. ${ }^{2}$ In medical applications, this could manifest as a clinically significant power loss. In addition, while the laser in general operates in a fundamental mode with regard to its spatial extent and intensity distribution for the laser beam, through bending higher order mode structures can be excited that can lead to slight intensity variations across the spatial extent of the beam/cross-section of the laser light.

We found a statistically significant difference in the peak power delivery in both the 200 and $365 \mu \mathrm{m}$ delivery fibers bent at radii ranging as low as $0.5 \mathrm{~cm}$ for the $200 \mu \mathrm{m}$ fiber and $1.7 \mathrm{~cm}$ for the $365 \mu \mathrm{m}$ fiber. The maximum power reduction recorded was $2.6 \%$ when the $200 \mu \mathrm{m}$ fiber was wrapped 10 times around a $1.3 \mathrm{~cm}$ radius. When bent one time around a radius of $0.5 \mathrm{~cm}$, the most extreme condition likely to be achieved in the operating room, our data showed a $1.25 \%$ power reduction as compared with baseline.

Following these results, we can conclude that under clinically achievable conditions there will be no clinically significant difference in power delivery or increase in laser propagation loss from bending alone. This result may not hold if the fiber is damaged or broken due to a sharp bend. Notably, the lack of clinically significant power loss was consistent to the smallest radius and highest number of turns the fiber could reliably undergo without breakage. These conditions exceeded the degree of bending that would be experienced by delivery fibers under clinical conditions.

For ureteroscopic surgical applications the demonstrated power changes are not expected to significantly influence the procedure. As surgeons operate under less taxing bending conditions than those explored in this study, endourologists can trust that laser output will be approximately stable during ureteroscopy regardless of stone location. As a consequence, flexible ureteroscopy remains an attractive option for management of inferior pole kidney stones.

There exists substantial literature in the field of optics and laser science regarding the influence of fiber curvature and bend radius on propagation losses; however, there is comparatively little data on the clinical impact of these known effects. Nazif and colleagues ${ }^{8}$ discussed some of the principles behind bending-induced propagation loss in a practical guide for urologists. However, within this discussion they did not delve into the clinical impact of these known effects. ${ }^{7}$

A study by Duchamp in 2012 differentiates the power outputs of a series of different laser fibers, classified by cladding and fiber diameter. To replicate clinical conditions, the experimental setup involved bending the delivery fibers to a small radius. The study did find variations in power outputs between different fiber types, although it did not achieve clinical significance for most fiber types assessed. There was one fiber type, which transmitted potentially clinically significantly lower power during their experimental testing phase. Although the primary variable of this study was not fiber bending radius as in ours, it did replicate some element of our bending conditions and explored power loss in a similar fashion. ${ }^{9}$

A closely related topic, the contribution of bending radius to fiber failure, has been significantly explored within the literature. Haddad and colleagues noted differences in the propensity of delivery fibers to fracture based on fiber size, 
curve diameter, and laser-firing settings. ${ }^{10}$ There was no direct exploration of power losses before complete fiber failure. This same concept was explored by Knudsen and colleagues in a study differentiating common delivery fibers by their propensity to break under bending conditions when fired continuously. ${ }^{11}$

The presented study was conducted under lower power settings since the photodetectors are sensitive to power (their damage threshold is below surgical power settings), which made it possible to trace the evolution of each individual pulse on the oscilloscope. While a direct detection of the output power from the fiber with a power meter could have been used with higher power settings, such a measurement generally only provides averaged power values over a certain time period. The high sensitivity in our experiments lends itself to precise measurements. Under the assumption that the observed bending behavior scales with power, we can therefore comfortably extrapolate our results to power levels more consistent with clinical practice. However, holmium lasers used in urological settings have outputs ranging from 30 to $120 \mathrm{~W}$. Future experimentation and validation could involve the use of higher power settings and of lasers rated for $>30 \mathrm{~W}$ of output.

Another foreseeable criticism of our study design was the placement of the bend in our delivery fiber. In endourological kidney stone management, the delivery fiber is subjected to its sharpest turns near the fiber tip. In our experimental setup (Fig. 1), a central portion of the fiber was bent around the measured cylinders. This technique enhanced reproducibility and reduced experimental error by minimizing any movement of the delivery fiber tip in relation to other experimental apparatuses. Since propagation loss is a linear phenomenon, the impact of bending should theoretically be the same regardless of bend location.

Additionally, during endourologic procedures delivery fibers are often bent and straightened repeatedly, oftentimes even before procedure start on the surgical table. In this experiment, the delivery fiber was only moved from the baseline position to each of bending radii once. Given the role that microscopic damage due to bending stress can theoretically play in power loss, it could be the case that repeated bending does lead to a clinically significant power loss, which was not appreciated under the experimental conditions. This possibility was not assessed and provides one potential avenue for further exploration.

The use of branded, stripped delivery fibers may affect the broader applicability of our results. Stripped laser fibers may in theory undergo structural changes during the stripping process; however, all trials were conducted with stripped fibers, maintaining internal validity. Additionally, there is potentially significant variability between the physical properties of delivery fibers from different companies, and even between fiber production lots.

Lastly, this experiment tested each fiber under each bending condition for an 8-second period of continuous firing. Clinically, fibers are subject to substantially longer periods of firing, which could directly damage the fiber cladding. Our experiment sought to measure exclusively the impact of bending radius on power transmission, but there is theory and evidence to suggest that bending fibers causes them to be more vulnerable to damage over extended periods of laser firing. ${ }^{2}$
Beyond using higher power settings and assessing the effects of repeated bending, a myriad of other future avenues of research have been suggested by our experiment and results. Although our data suggest no clinically relevant power loss from bending alone over short-term use, other aspects of the clinical scenario could affect power output: repetitive use of the fiber over the course of multiple hours and repeated bending of the fiber are two such examples.

\section{Conclusion}

We assessed the impact of fiber bending radius on the delivered power at the end of the fiber using a highly accurate oscilloscope, a urological holmium laser, and commonly used large-core optical fibers. We believe this experimental model adequately assesses the potential for fiber bending to affect endourological surgery. By bending the fiber in excess of any clinically achievable conditions, the phenomenon has been robustly explored. A minimal, statistically significant reduction in power output was noted under certain bending conditions. No clinically significant power losses were appreciated under any bending condition across either 200 or $365 \mu \mathrm{m}$ fibers.

Our results provide a novel analysis of the impact of a specific intraoperative variable on clinical practice. There is no evidence to suggest that clinically achievable bending radii alone have an appreciable impact on end-fiber power delivery during flexible ureteroscopy.

\section{Acknowledgment}

The authors would like to thank Lumenis ${ }^{\odot}$ (Yokneam Illit, Israel) for the use of their holmium laser in this experiment.

\section{Author Disclosure Statement}

No competing financial interests exist.

\section{Funding Information}

No funding was received for this article.

\section{References}

1. Parsons RL, Campbell JL, Thomley MW, Butt CG, Gordon TE Jr. The effect of the laser of dog bladders: A preliminary report. J Urol 1966;95:716-717.

2. Floratos DL, de la Rosette JJ. Lasers in urology. BJU Int 1999;84:204-211.

3. Knudsen BE, Glickman RD, Stallman KJ, Maswadi S, Chew BH, Beiko DT, Denstedt JD, Teichman JMH. Performance and safety of holmium:YAG laser optical fibers. J Endourol 2005;19:1092-1097.

4. Mues AC, Teichman JMH, Knudsen BE. Evaluation of 24 holmium:YAG laser optical fibers for flexible ureteroscopy. J Urol 2009;182:348-354.

5. Khemees TA, Shore DM, Antiporda M, Teichman JMH, Knudsen BE. Evaluation of a new 240- $\mu \mathrm{m}$ single-use holmium:YAG optical fiber for flexible ureteroscopy. J Endourol 2013;27:475-479.

6. Méndez A. Optics in medicine. In: Al-Amri M, El-Gomati M, Zubairy M, eds. Optics in Our Time. Cham: Springer, 2016, pp. 299-333. 
7. Semenkoff M. Bending effect on light propagation in an optical fiber: Application to a temperature sensor. Opt Lasers Eng 1992;17:179-186.

8. Nazif OA, Teichman JMH, Glickman RD, Welch AJ. Review of laser fibers: A practical guide for urologists. J Endourol 2004;18:824.

9. Duchamp JG. Evaluation of holmium:YAG laser optical fibers for flexible ureteroscopy using a relevant Benchtop model. EndoBeam ${ }^{\mathrm{TM}}$ White Paper, 2012.

10. Haddad M, Emiliani E, Rouchausse Y, et al. Impact of the curve diameter and laser settings on laser fiber fracture. J Endourol 2017;31:918-921.

11. Knudsen BE, Glickman RD, Teichman JM. A comparison of single use and reusable small core sized holmium:YAG laser fibers: Is there a difference in the risk of fiber failure? Proceedings of SPIE-The International Society for Optical Engineering, 2006.
Address correspondence to:

Dylan Heckscher, $A B$

Department of Urology

Boston University School of Medicine

725 Albany Street, Suite 3B

Boston, MA 02118

USA

E-mail: dylanh@bu.edu

\section{Abbreviations Used \\ RMS = root-mean-square \\ $\mathrm{au}=$ arbitrary unit}

